PHYSICAL REVIEW D 94, 069901(E) (2016)

\title{
Erratum: IceCube neutrinos, decaying dark matter, and the Hubble constant [Phys. Rev. D 92, 061301(R) (2015)]
}

Luis A. Anchordoqui, Vernon Barger, Haim Goldberg, Xing Huang, Danny Marfatia, Luiz H. M. da Silva, and Thomas J. Weiler (Received 25 August 2016; published 7 September 2016)

DOI: 10.1103/PhysRevD.94.069901

Reference [1] was revised after our paper was published. The dark-matter decay width required to reconcile the tension in the determination of the Hubble constant was reduced by 2 orders of magnitude. We update our analysis accordingly and account for the new analysis of Ref. [2] that incorporates lensing constraints. For $f_{X} \simeq 0.05$ and $\Gamma_{X} \simeq 5000 \mathrm{~km} \mathrm{~s}^{-1} \mathrm{Mpc}^{-1}$, the best-fit parameters are $\tau_{X} \simeq 6 \times 10^{15} \mathrm{~s}, \quad m_{X} \simeq 76 \mathrm{PeV}, \quad Y_{X} \simeq 2.8 \times 10^{-19}, \quad \omega_{\nu} \simeq 2.6 \times 10^{-11} \mathrm{eV} \mathrm{cm}^{-3}$, and $\mathcal{B}_{X \rightarrow \nu \bar{\nu}} \sim 3 \times 10^{-9}$. For this set of parameters, Fig. 1 and the ensuing conclusions remain unchanged.

[1] Z. Berezhiani, A. D. Dolgov, and I. I. Tkachev, Phys. Rev. D 92, 061303(R) (2015).

[2] A. Chudaykin, D. Gorbunov, and I. Tkachev, Phys. Rev. D 94, 023528 (2016). 